\title{
Resistencia bacteriana y factores asociados en pacientes con pie diabético infectado sin desenlace de amputación mayor en un hospital nacional peruano Bacterial resistance and associated factors in patients with infected diabetic foot with no major amputation outcomes in a Peruvian national hospital
}

Correspondencia

Marlon Augusto Yovera Aldana marlonyovera@hotmail.com

\section{Recibido: 14/08/2017}

Arbitrado por pares

Aprobado: 13/09/2017

Citar como: Yovera-Aldana $M$, Rodríguez A, Vargas $M$, Heredia $P$, Huamán MO, Vargas-Vilca J, et al. Resistencia bacteriana y factores asociados en pacientes con pie diabético infectado sin desenlace de amputación mayor en un hospital nacional peruano. Acta Med Peru. 2017;34(3):173-81
Marlon Yovera-Aldana ${ }^{1, a}$, Antuané Rodríguez ${ }^{2, b}$, Mariela Vargas ${ }^{2, b}$, Paula Heredia2,b, Manuel O. Huamán²,b, Jesús Vargas-Vilca²,b, Claudia Yalán²,b, Eduardo García-Orbegoso 3 ,a

1 Escuela de Medicina, Universidad Nacional de Piura. Piura, Perú.

2 Instituto de Investigación en Ciencias Biomédicas, Universidad Ricardo Palma Lima, Perú.

3 Servicio de Endocrinología, Hospital Nacional Edgardo Rebagliati Martins. Lima, Perú.

a Médico Endocrinólogo, b Estudiante de Medicina

\section{RESUMEN}

Objetivo: Determinar la resistencia bacteriana y factores asociados en pacientes con pie diabético infectado sin desenlace de amputación mayor. Materiales y métodos: Estudio transversal, analítico, de revisión de historias clínicas de pacientes con pie diabético infectado del 2010-2014 en el Hospital Nacional Edgardo Rebagliati Martins. Según protocolo, las muestras de cultivo consistieron en tejidos que se obtuvieron después de desbridar la zona afectada. Sólo se usaron medios aerobios. Resultados: Se incluyeron 88 pacientes, aislándose 128 bacterias. El 81,8\% fue varón con edad promedio de 60,6 años. El 76\% tenía tiempo de diabetes $>10$ años y el $18,7 \%$ presentó $\mathrm{Hba1c}<7,0 \%$. Según clasificación Wagner, $39,8 \%$ fue grado 3 y $40,9 \%$ grado 4 . La infección grado moderada, según IDSA, fue la más frecuente $(80,7 \%)$, al igual que la isquemia moderada $(65,9 \%)$ y $44 \%$ tuvo infección previa por pie diabético. El $42 \%$ de los cultivos fue polimicrobiano, las bacterias aisladas más frecuentes fueron Escherichia coli (23,4\%); Enterococcus faecalis (14,1\%) y Staphylococcus aureus (13,3\%). El 33\% de las Enterobacterias fueron productores de betalactamasa de Espectro Extendido (BLEE). Se halló asociación de BLEE con infección previa por pie diabético, mayor PCR y fascitis necrotizante por LRINEC. EI 71\% de los S. aureus fue meticilino resistente (SAMR), hallándose asociación sólo con PCR alto. Conclusiones: En pacientes con pie diabético sin desenlace de amputación mayor, se encontró una elevada frecuencia de Enterobacterias BLEE positivas y SAMR. Infección previa, PCR alto y LRINEC >8 estuvo asociado a la presencia de BLEE y sólo PCR alto a SAMR.

Palabras clave: Pie diabético; Farmacorresistencia; Infecciones bacterianas (fuente: DeCS BIREME). 


\begin{abstract}
Objective: To determine bacterial resistance and its associated factors in patients with infected diabetic foot with no major amputation outcomes. Materials and methods: This is a cross-sectional analytical study, which consisted in reviewing clinical records from patients with infected diabetic foot seen during the period 2010-2014 in the Edgardo RebagliatiMartins Hospital. According to the protocol, samples for culture consisted in tissues that were obtained after debriding the affected areas. Only aerobic media for cultures were used. Results: Eighty-eight patients were included, and 128 bacterial isolates were obtained. Most of the subjects (81.8\%) were male, and their average age was 60.6 years. Three-fourths of all patients $(76 \%)$ had diabetes for more than 10 years, and $18.7 \%$ had $\mathrm{Hba} 1 \mathrm{c}<7.0 \%$. According to the Wagner classification, $39.8 \%$ were grade 3 and $40.9 \%$ were grade 4 . Moderated grade infections, according to the IDSA, were the most frequent instances $(80.7 \%)$, as well as moderate ischemia $(65.9 \%)$, and $44 \%$ of patients had a previous infection on their diabetic foot. Only $42 \%$ of all cultures were polymicrobial, and most frequently isolated microorganisms were Escherichia coli (23.4\%), Enterococcus faecalis (14.1\%), and Staphylococcus aureus (13.3\%). One-third (33\%) of Enterobacteriaceae were Extended-Spectrum Beta-lactamase (ESBL) producers. There was an association of having ESBL microorganisms with previous diabetic foot infections, higher CRP values and necrotizing fasciitis according to LRINEC. Most S. aureus strains (71\%) were methicillin-resistant (MRSA), and this was associated with high CRP values. Conclusions: In patients with diabetic foot with no major amputation outcomes, there was a high frequency of ESBL Enterobacteriaceae and MRSA. The occurrence of a previous infection, high CRP values and LRINEC $>8$ were associated with the presence of ESBL, and only high CRP values were associated with MRSA.
\end{abstract}

Keywords: Diabetic Foot; Drug resistance; Bacterial infections (source: MeSH NLM).

\section{INTRODUCCIÓN}

La resistencia bacteriana es un fenómeno que se incrementa en todo el mundo, principalmente en países en desarrollo. Su progresiva presencia en los casos de pie diabético infectado, aumenta más los costos ya elevados de atención, prolonga estancias hospitalarias y eleva los porcentajes de ocupación ${ }^{[1]}$. La lesión se inicia con una úlcera ocasionada por la interacción de neuropatía diabética, deformidad biomecánica y/o la enfermedad arterial periférica; la úlcera está presente previamente en el 40 a $70 \%$ de las infecciones que acuden a hospitalización, y si esta es si es severa, se convierte en la principal causa de amputación no traumática de miembros inferiores a nivel mundial, impactando en la vida de la persona al ocasionar ausentismo laboral, carga social y menor expectativa de vida ${ }^{[2,3]}$

Existen múltiples factores que intervienen en el tratamiento de las infecciones del pie diabético, como la sospecha de las bacterias involucradas, la terapia antibiótica previa recibida, la terapia empírica estándar recomendada; la severidad de la infección, la orientación con el cultivo y antibiograma obtenido y factores personales que modifican la farmacocinética y farmacodinamia habitual ${ }^{[4]}$.

Los reportes de susceptibilidad microbiana locales juegan un rol importante para proveer información de la ocurrencia y diseminación de la resistencia bacteriana, definir lineamientos de terapia empírica y medidas de control. El Instituto Nacional de Salud (INS) del Perú realiza esta vigilancia permanente, aunque los informes no son específicos para infecciones del pie diabético ${ }^{[6]}$.
El Enterococcus faecium resistente a vancomicina (ERV), el Staphylococcus aureus meticilino resistente (SAMR), la Klebsiella pneumoniae productora de betalactamasa de espectro extendido (BLEE), el Acinetobacter baumannii, la Pseudomonas aeruginosa y las Enterobacterias, agrupadas bajo el acrónimo ESKAPE, son calificados por la International Disease Society American (IDSA) como patógenos de alta prioridad de reporte y seguimiento por representar un gran problema clínico y de salud pública, al tener limitadas alternativas terapéuticas y pronósticos sombríos ${ }^{[5]}$.

El Hospital Nacional Edgardo Rebagliati Martins (HNERM), hospital dependiente del Seguro Social de Salud (EsSalud), instauró desde 1997 un tratamiento intensivo del pie diabético infectado, que incluye desbridamiento quirúrgico inmediato continuo, terapia antibiótica endovenosa, nutrición balanceada, insulinoterapia revascularización si se determinaba necesario [7], logrando disminuir la frecuencia de amputación mayor pero incrementando la demanda y el tiempo de tratamiento de los pacientes. Ya que es importante conocer el perfil bacteriológico de estos sujetos, se propuso determinar la resistencia bacteriana antibiótica y factores asociados en pacientes con pie diabético infectado que no culminaron en amputación mayor del HNERM.

\section{MATERIALES Y MÉTODOS}

Se realizó un estudio transversal-analítico de los pacientes con pie diabético infectado sin desenlace de amputación mayor que asistieron al área de pie diabético del HNERM durante el periodo 2010-2014. Se seleccionaron los registros clínicos de los pacientes que tenían cultivo y antibiograma tomados dentro 
Tabla 1.Características generales de pacientes con pie diabético infectado sin desenlace de amputación mayor del Hospital Nacional Edgardo Rebagliati Martins.

\begin{tabular}{|c|c|}
\hline Características & n (\%) \\
\hline \multicolumn{2}{|l|}{ Edad } \\
\hline$<60$ años & $39(44,3)$ \\
\hline $61-70$ años & $30(34,1)$ \\
\hline$>70$ años & $19(21,6)$ \\
\hline \multicolumn{2}{|l|}{ Sexo } \\
\hline Masculino & $72(81,8)$ \\
\hline \multicolumn{2}{|l|}{ Tiempo de diabetes } \\
\hline$<10$ años & $21(23,8)$ \\
\hline $10-20$ años & $32(36,4)$ \\
\hline > 20 años & $35(39,8)$ \\
\hline \multicolumn{2}{|l|}{ Hemoglobina glicosilada } \\
\hline$<7 \%$ & $14(18,7)$ \\
\hline $7-9 \%$ & $29(38,7)$ \\
\hline$>9 \%$ & $32(42,6)$ \\
\hline \multicolumn{2}{|l|}{ Clasificación Wagner } \\
\hline 2 & $16(18,2)$ \\
\hline 3 & $35(39,8)$ \\
\hline 4 & $36(40,9)$ \\
\hline 5 & $1(1,1)$ \\
\hline \multicolumn{2}{|l|}{ Localización } \\
\hline Ante pie & $38(43,2)$ \\
\hline Medio pie & $39(44,4)$ \\
\hline Retro pie & $11(12,5)$ \\
\hline \multicolumn{2}{|l|}{ Infección IDSA } \\
\hline Grado 2: Leve & $10(11,4)$ \\
\hline Grado 3 :Moderada & $71(80,7)$ \\
\hline Grado 4 : Severa & $7(7,9)$ \\
\hline Infección pie diabético previa & $39(44,3)$ \\
\hline \multicolumn{2}{|l|}{ Enfermedad arterial periférica } \\
\hline Leve & $26(29,6)$ \\
\hline Moderada & $58(65,9)$ \\
\hline Severa & $4(4,5)$ \\
\hline Proteína $\mathrm{C}$ reactiva (>15 g/dL) & $19(23,8)$ \\
\hline \multicolumn{2}{|l|}{ Leucocitos } \\
\hline$<15 \times 10^{3}$ & $56(63,6)$ \\
\hline $15-25 \times 10^{3}$ & $26(29,6)$ \\
\hline$>25 \times 10^{3}$ & $6(6,8)$ \\
\hline \multicolumn{2}{|l|}{ Hemoglobina } \\
\hline$>13,5 \mathrm{~g} / \mathrm{dL}$ & $2(2,3)$ \\
\hline $11-13,5 \mathrm{~g} / \mathrm{dL}$ & $31(35,2)$ \\
\hline$<11 \mathrm{~g} / \mathrm{dL}$ & $55(62,5)$ \\
\hline Creatinina (> 1,6 mg/dL) & $14(15,9)$ \\
\hline Sodio (<135 mg/dL) & $35(40,2)$ \\
\hline Glucosa (> $180 \mathrm{mg} / \mathrm{dL}$ ) & $47(53,4)$ \\
\hline Fascitis necrotizante por LRINEC (Puntaje $\geq 8$ ) & $22(25,0)$ \\
\hline \multicolumn{2}{|l|}{ Número de bacterias en cultivo } \\
\hline Sin aislamiento & $3(3,4)$ \\
\hline Monomicrobiano & $48(54,6)$ \\
\hline Polimicrobiano & $37(42,0)$ \\
\hline 2 bacterias & 30 \\
\hline 3 bacterias & 6 \\
\hline 4 bacterias & 1 \\
\hline
\end{tabular}

IDSA: International Disease Society American; LRINEC: Laboratory Risk Indicator for necrotizing fascitis. de la primera semana de infección, ecografía doppler arterial y datos bioquímicos completos.

Todos los pacientes recibieron desbridamiento quirúrgico precoz ambulatorio (DQPA), procedimiento estándar en el Servicio de Endocrinología del HNERM, que consiste en la escisión de tejidos no viables (piel, tejido celular subcutáneo, músculo, tendones y hueso) más drenaje manual de secreciones purulentas-, en sala de procedimientos con una frecuencia mínima de 3 veces por semana, realizada desde el primer contacto por un médico endocrinólogo con amplia experiencia en pie diabético. Los pacientes en general permanecen hospitalizados en promedio dos semanas, y luego continúan sus curaciones en forma ambulatoria hasta el completo cierre de la herida, evaluando también potenciales recaídas y/o recurrencias. Utilizando para la limpieza de la herida suero salino y como cobertura, gasa simple.

Las muestras para cultivo se tomaron de zonas no expuestas del lecho de la herida luego del desbridamiento quirúrgico inicial, pudiendo ser tejido celular subcutáneo, músculo, tendón o hueso; colocados en frascos estériles y conducidos al laboratorio de microbiología en el lapso de dos horas. Se usó el sistema automatizado de identificación bacteriana y estudio de sensibilidad antimicrobiana VITEK 2 (Laboratorio bioMérieux, Argentina). No se evaluaron cepas anaerobias. Se definió como BLEE aquellas enterobacterias resistentes a todos los antibióticos betalactámicos excepto los carbapenémicos, cefamicinas y combinaciones de betalactámicos con inhibidores de betalactamasas, como el tazobactam y el sulbactam según lo planteado por Bush-Jacoby-Madeiros ${ }^{[8]}$. Se consideró SAMR al S. aureus con resistencia a oxacilina ${ }^{[6]}$. Se consideró resistencia antibiótica a los resultados "Resistente" e "Intermedio" del informe de sensibilidad.

Tabla 2. Perfil bacteriológico de pacientes con pie diabético infectado sin desenlace de amputación mayor del Hospital Nacional Edgardo Rebagliati Martins.

\begin{tabular}{lccc} 
Características & $\begin{array}{c}\mathbf{N}^{\circ} \text { de } \\
\text { bacterias } \\
\text { (n=128) }\end{array}$ & $\begin{array}{c}\text { \% } \\
\text { total }\end{array}$ & $\begin{array}{c}\% \\
\text { parcial }\end{array}$ \\
\hline Bacterias gramnegativas & 89 & 69,5 & 100 \\
Escherichia coli & 30 & 23,4 & 33,7 \\
Proteus mirabilis & 13 & 10,2 & 14,6 \\
Klebsiella pneumoniae & 10 & 7,8 & 11,2 \\
Morganella morganii & 9 & 7,0 & 10,1 \\
Citrobacter freundii & 7 & 5,5 & 7,8 \\
Pseudomonas aeruginosa & 6 & 4,7 & 6,7 \\
Enterobacter cloacae & 5 & 3,9 & 5,6 \\
Acinetobacter baumannii & 3 & 2,3 & 3,3 \\
Proteus vulgaris & 2 & 1,6 & 2,2 \\
Providencia sp & 2 & 1,6 & 2,2 \\
Serratia marcescens & 1 & 0,8 & 1,1 \\
Stenotrophomonas maltophilia & 1 & 0,8 & 1,1 \\
Bacterias grampositivas & 39 & 30,5 & 100 \\
Enterococcus faecalis & 18 & 14,1 & 46,2 \\
Staphylococcus aureus & 17 & 13,3 & 43,6 \\
Streptococcus sp & 4 & 3,1 & 10,2 \\
\hline
\end{tabular}


Tabla 3. Porcentajes de resistencia bacteriana en pacientes con pie diabético infectado sin desenlace de amputación mayor del Hospital Edgardo Rebagliati Martins

\begin{tabular}{|c|c|c|c|c|c|c|c|c|c|c|c|c|}
\hline \multirow{2}{*}{ Antibiótico } & \multicolumn{9}{|c|}{ Bacteria gramnegativa } & \multicolumn{3}{|c|}{ Bacteria grampositiva } \\
\hline & EC & PM & PV & KP & MM & CF & $\mathrm{ECl}$ & PA & $\mathrm{AB}$ & EF & SA & SP \\
\hline \multicolumn{13}{|l|}{ Betalactámicos } \\
\hline Penicilina & -- & -- & -- & -- & -- & -- & -- & -- & -- & 12 & 100 & 0 \\
\hline Oxacilina & -- & -- & -- & -- & -- & -- & -- & -- & -- & -- & 71 & -- \\
\hline Ampicilina & 89 & 100 & 0 & 89 & 100 & 100 & 100 & -- & -- & 6 & 100 & 0 \\
\hline AMX/CLA & 36 & 31 & 0 & 67 & 100 & 100 & 100 & -- & -- & 0 & 100 & 0 \\
\hline TIC / CLA & 19 & 0 & 0 & 50 & 100 & 100 & 100 & 100 & 100 & -- & -- & -- \\
\hline PIP/ TAZ & 0 & 0 & 0 & 25 & 100 & 100 & 80 & 83 & 100 & -- & -- & -- \\
\hline Aztreonam & 66 & 31 & -- & 78 & 100 & 100 & 100 & 100 & -- & -- & -- & -- \\
\hline \multicolumn{13}{|l|}{ Cefalosporinas } \\
\hline Cefalotina & 57 & 33 & -- & 100 & -- & 100 & -- & -- & -- & 100 & 100 & 0 \\
\hline Cefazolina & 68 & 82 & 100 & 75 & 100 & 100 & 100 & -- & -- & 100 & -- & -- \\
\hline Cefuroxima & 64 & 31 & 100 & 88 & 100 & 100 & 100 & -- & -- & 100 & 100 & 0 \\
\hline Cefoxitina & 7 & 15 & 0 & 0 & 100 & 100 & 100 & -- & -- & 100 & 100 & 0 \\
\hline Cefotaxima & 67 & 31 & 0 & 75 & 100 & 100 & 100 & 100 & 100 & 100 & 50 & 0 \\
\hline Ceftriaxona & 69 & 31 & 0 & 78 & 100 & 83 & 100 & 100 & 100 & 100 & 100 & 0 \\
\hline Ceftazidima & 63 & 33 & 0 & 78 & 100 & 100 & 100 & 83 & 100 & 100 & 100 & 0 \\
\hline Cefepime & 62 & 31 & 0 & 71 & 11 & 14 & 40 & 50 & 100 & 100 & 80 & 0 \\
\hline \multicolumn{13}{|l|}{ Carbapenémicos } \\
\hline Ertapenem & 0 & 0 & 0 & 0 & 0 & 0 & 0 & -- & -- & -- & -- & -- \\
\hline Imipenem & 4 & 0 & 0 & 0 & 0 & 0 & 0 & 83 & -- & -- & -- & -- \\
\hline Meropenem & 0 & 0 & 0 & 0 & 0 & 0 & 0 & 83 & 100 & -- & -- & -- \\
\hline \multicolumn{13}{|l|}{ Glucopéptidos } \\
\hline Teicoplanina & -- & -- & -- & -- & -- & -- & -- & -- & -- & 0 & 0 & -- \\
\hline Vancomicina & -- & -- & -- & -- & -- & -- & -- & -- & -- & 0 & 0 & -- \\
\hline \multicolumn{13}{|l|}{ Aminoglicósidos } \\
\hline Amikacina & 0 & 8 & 0 & 11 & 11 & 0 & 25 & 17 & 100 & -- & 50 & -- \\
\hline Gentamicina & 48 & 46 & 0 & 56 & 22 & 43 & 40 & 50 & 100 & 63 & 50 & -- \\
\hline \multicolumn{13}{|l|}{ Quinolonas } \\
\hline Ciprofloxacino & 90 & 77 & 0 & 89 & 67 & 57 & 50 & 83 & 100 & 61 & 93 & -- \\
\hline Levofloxacino & 86 & 46 & -- & 44 & 56 & 20 & 40 & 67 & 100 & 57 & 83 & 33 \\
\hline \multicolumn{13}{|l|}{ Otros } \\
\hline Eritromicina & -- & -- & -- & -- & -- & -- & -- & -- & -- & 88 & 81 & 0 \\
\hline Clindamicina & -- & -- & -- & -- & -- & -- & -- & -- & -- & 50 & 82 & 0 \\
\hline Rifampicina & -- & -- & -- & -- & -- & -- & -- & -- & -- & 53 & 20 & -- \\
\hline Tetraciclina & 79 & 100 & 50 & 89 & 100 & 80 & 50 & -- & 100 & 81 & 50 & 100 \\
\hline Cotrimoxazol & 83 & 85 & 0 & 89 & 100 & 71 & 60 & -- & 100 & 50 & 27 & -- \\
\hline
\end{tabular}

EC: Escherichia coli; PM: Proteus mirabilis; KP: Klebsiella pneumoniae: MM: Morganella morganii; CF: Citrobacter freundii: PA: Pseudomonas aeruginosa, ECl: Enterobacter cloacae; AB: Acinetobacter baumannii; EF: Enterococcus faecalis; SA: Staphylococcus aereus; SP: Streptococcus sp.; AMX/CLA: amoxicilina/ácido clavulánico; TIC/CLA: ticarcilina/ácido clavulánico; PIP/TAZ: piperacilina /tazobactam 
Tabla 4. Resistencias bacterianas de importancia epidemiológica en pacientes con pie diabético infectado sin desenlace de amputación mayor del Hospital Nacional Edgardo Rebagliati Martins.

\begin{tabular}{lcc} 
& \multicolumn{1}{c}{ Tipo de resistencia } & Proporción \\
\hline Staphylococcus aureus meticilino resistente & $12 / 17$ & 71 \\
Staphylococcus aureus resistente a vancomicina & $0 / 17$ & 0 \\
Enterococcus resistente a vancomicina & $0 / 18$ & 0 \\
Enterobacterias BLEE (+) & $26 / 80$ & 33 \\
$\quad$ Escherichia coli & $17 / 30$ & 57 \\
Proteus mirabilis & $4 / 13$ & 31 \\
Klebsiella pneumoniae & $4 / 10$ & 40 \\
Proteus vulgaris & $1 / 2$ & 50 \\
Enterobacteria BLEE (+) resistente a carbapenem & $1 / 26$ & 4 \\
Pseudomonas aeruginosa resistente a carbapenem & $5 / 6$ & 83 \\
Pseudomonas aeruginosa resistente a betalactámico, quinolona y aminoglicósido & $1 / 6$ & 17 \\
Acinetobacter baumannii pandrogoresistente & $3 / 3$ & 100 \\
\hline
\end{tabular}

* Porcentaje relativo al total de casos por cada tipo. Por ejemplo: $71 \%$ resulta del cociente de 12 casos resistentes sobre el total de S. aureus.

Se consignaron las variables edad, sexo, tiempo de diagnóstico de diabetes, grado de clasificación Wagner, severidad de infección según IDSA, antecedente de infección por pie diabético, localización de úlcera pedia, grado de enfermedad arterial periférica según patrón de flujo arterial de miembros inferiores por ecografía doppler (trifásica, bifásica o monofásica), hemoglobina glicosilada de los tres últimos meses previo a la evaluación y bioquímica de ingreso (glucosa, proteína C reactiva $[P C R]$, leucocitos, hemoglobina, sodio, creatinina). Se calculó el Laboratory Risk Indicator Necrotizing Fascitis (LRINEC) para reconocimiento temprano de fascitis necrotizante ${ }^{[9]}$.

Los antibióticos analizados fueron aquellos del Petitorio Nacional Único de Medicamentos Esenciales de la Dirección General de Medicamentos Insumos y Drogas del Ministerio de Salud del Perú y de EsSalud ${ }^{[5]}$.

Los datos fueron analizados en el programa STATA 11.1. Se describió la frecuencia de cada bacteria según el total y según pertenencia a gramnegativo o grampositivo. Se calculó el porcentaje de resistencia de cada bacteria según antibiótico. Se determinó la frecuencia de resistencia de importancia clínica. Se describió la resistencia bacteriana en enterobacterias BLEE positivas, BLEE negativas y de SAMR positivos y SAMR negativos. Se analizó los factores asociados a enterobacterias BLEE (+) y factores asociados a SAMR (+). Para variables categóricas se analizó con Chi cuadrado o $\mathrm{F}$ exacta de Fisher si los valores esperados eran $>5$ en más del $25 \%$ de las cuadrículas. Para variables cuantitativas se usó T de Student o U de Mann Whitney según evaluación de normalidad de la distribución.

El proyecto tuvo la aprobación del Comité Científico y Ético del Hospital Nacional Edgardo Rebagliati Martins.

\section{RESULTADOS}

De los 210 registros de pacientes salvados de amputación mayor del 2010 al 2014, se incluyeron a 88 que cumplían con los criterios de inclusión. El promedio de edad fue de $60,6 \pm 12$ años y el $81,8 \%$ era del sexo masculino. La mediana del tiempo de diabetes fue de 15 años con una $\mathrm{HbA} 1 \mathrm{c}$ de 8,5\%.

En el grupo estudiado, predominaron los grados 3 y 4 de la escala Wagner (39,8 y 40,9\%, respectivamente). La zona más afectada fue la región del medio pie $(44,4 \%)$, seguida del ante pie $(43,2 \%)$. El 80,7\% tuvo una severidad moderada de infección según IDSA, el $65,9 \%$ presentaba enfermedad periférica moderada (ondas bifásicas), el $25 \%$ presentó fascitis necrotizante (puntaje $\geq 8$ en escala LRINEC ${ }^{[8]}$ y el $44 \%$ tuvo infección previa por pie diabético. (Tabla 1).

El 42\% de los cultivos realizados indicó la presencia de más de una bacteria (solo cepas aeróbicas); el promedio fue de 1,5 bacterias por cultivo (Tabla 1).

De las 128 bacterias aisladas, el 30,5\% fueron bacterias grampositivas y $69,5 \%$ bacterias gramnegativas. Las bacterias más comunes fueron E. coli $23,4 \%$; E. faecalis $14,1 \%$; S. aureus $13,3 \%$ y $P$. mirabilis $10,2 \%$ (Tabla 2 ).

De las Enterobacterias, se hallaron 26 bacterias BLEE positivas (33\%) y en ellas, sólo 1 (4\%) tuvo de resistencia a imipenem/ cilastatina. Se halló alta resistencia a carbapenémicos en las bacterias no fermentadoras: P. aeruginosa del $83 \%$ y $A$. baumannii 100\% (Tablas 3 y 4). Las Enterobacterias BLEE negativas tuvieron una resistencia del $89,4 \%$ a ciprofloxacino y de $56 \%$ a ceftriaxona como fármacos de primera línea de las guías clínicas de tratamiento (Tabla 5). 
Tabla 5. Porcentajes de resistencia bacteriana de Enterobacterias $\operatorname{BLEE}(+)$, Enterobacterias BLEE (-), SAMR (+) y SAMR (-) en pacientes con pie diabético infectado sin desenlace de amputación mayor del Hospital Nacional Edgardo Rebagliati Martins.

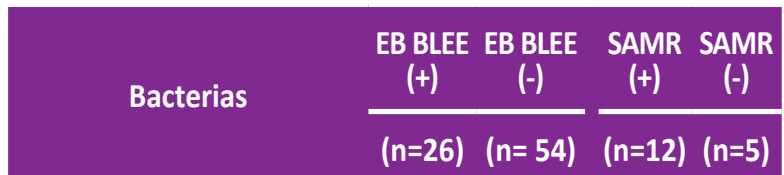

Betalactámicos

\begin{tabular}{|c|c|c|c|c|}
\hline Oxacilina & -- & -- & 100 & 0 \\
\hline Ampicilina & 100 & 92 & 100 & 100 \\
\hline Amoxicilina/ácido clavulánico & 40 & 67 & -- & -- \\
\hline Piperacilina/tazobactam & 4 & 49 & -- & -- \\
\hline Aztreonam & 96 & 58 & -- & -- \\
\hline \multicolumn{5}{|l|}{ Cefalosporinas } \\
\hline Cefoxitina & 4 & 53 & -- & -- \\
\hline Cefotaxima & 96 & 57 & -- & -- \\
\hline Ceftriaxona & 96 & 56 & -- & -- \\
\hline Ceftazidima & 96 & 59 & -- & -- \\
\hline Cefepime & 96 & 18 & -- & -- \\
\hline \multicolumn{5}{|l|}{ Carbapenémicos } \\
\hline Ertapenem & 0 & 0 & -- & -- \\
\hline Imipenem & 4 & 0 & -- & -- \\
\hline Meropenem & 0 & 0 & -- & -- \\
\hline Glicopéptidos & -- & -- & & \\
\hline Vancomicina & -- & -- & 0 & 0 \\
\hline \multicolumn{5}{|l|}{ Aminoglicósidos } \\
\hline Amikacina & 0 & 8 & -- & -- \\
\hline Gentamicina & 69 & 29 & -- & -- \\
\hline \multicolumn{5}{|l|}{ Quinolonas } \\
\hline Ciprofloxacino & 92 & 69 & 100 & 60 \\
\hline Levofloxacino & -- & -- & 100 & 0 \\
\hline \multicolumn{5}{|l|}{ Otros } \\
\hline Eritromicina & -- & -- & 92 & 60 \\
\hline Clindamicina & -- & -- & 89 & 60 \\
\hline Rifampicina & -- & -- & 25 & 0 \\
\hline Tetraciclina & 92 & 81 & 0 & 100 \\
\hline Cotrimoxazol & 89 & 75 & 33 & 0 \\
\hline
\end{tabular}

EB: Enterobacterias; BLEE: Betalactamasa de espectro extendido; SAMR: Staphylococcus aureus meticilino resistente.
De las bacterias grampositivas hubo un $71 \%$ de resistencia a oxacilina o SAMR. No hubo casos de resistencia a vancomicina en S. aureus o E. faecalis (Tabla 3 y 4). La resistencia en SAMR para ciprofloxacino y clindamicina fue de $100 \%$ y $89 \%$ respectivamente. En cambio, para S. aureus no meticilino resistente fue del $66,7 \%$ y $50 \%$ también respectivamente (Tabla 5).

Las Enterobacterias BLEE positivas, comparadas con BLEE negativas, se asociaron a infección de pie diabético previa, mayor proteína C reactiva y elevada frecuencia de LRINEC $\geq 6$ sugerente de fascitis necrotizante. Los SAMR comparado con aquellos no SAMR, sólo estuvieron asociados a PCR alto (Tabla 6).

\section{DISCUSIÓN}

La progresiva resistencia bacteriana exige sistemas de vigilancia para identificación de las bacterias y su susceptibilidad ${ }^{[6]}$. El elaborar y actualizar estos reportes, junto a otras intervenciones, en los establecimientos de salud que tratan pie diabético disminuirá el fracaso terapéutico, la resistencia antibiótica, la estancia hospitalaria y la tasa de amputación mayor.

Predominó el sexo masculino (80\%), como también en otra serie de úlceras pedias infectadas ${ }^{[10,11]}$, a diferencia de los estudios de pie con lesiones preulcerativas donde se halla más presencia de mujeres debido a su mayor rol previsor ${ }^{[12]}$. El $55 \%$ era mayor a 60 años, series pasadas mostraban un promedio mayor ${ }^{[11,13]}$; al contrario de las actuales que revelan complicaciones a más temprana edad ${ }^{[10,14]}$, es decir, afectará progresivamente a la población económicamente activa ${ }^{[3]}$. El $25 \%$ presentó lesión pedia con menos de 10 años de diabetes, incluso muchas complicaciones se encuentran presentes al momento del diagnóstico ${ }^{[10,13]}$. Sólo el $18,7 \%$ de los pacientes presentó una diabetes controlada (HbA1c menor a 7\%); si bien actualmente la meta es en función a la edad, tiempo de enfermedad, complicaciones, etc.; ésta fue menor con respecto a otra serie peruana ${ }^{[12]}$.

Un 80,4\% presentó un grado 3 y 4 según la clasificación Wagner. El 80,7\% presentó una infección moderado según IDSA, es decir eritema de más de $2 \mathrm{~cm}$ o absceso y un 7,9\% tenía repercusiones sistémicas como fiebre, hipotensión o trastorno de conciencia. Además, un $65,9 \%$ tenía enfermedad arterial periférica moderada y un $4,5 \%$ severa. Todas estas características, tienen mayor frecuencia y severidad que otras series peruanas y están asociadas a alto riesgo de amputación mayor; no obstante, sólo se les realizó drenaje de secreciones, desbridamiento de tejidos blandos y/o amputaciones menores ${ }^{[10,11]}$.

Considerando que sólo se tomaron cultivos para aerobios y de muestras de tejido (tendones, celular subcutáneo y hueso); un $42 \%$ de las infecciones fue polimicrobiana, mayor a otro estudio local donde reportó sólo $20 \%$ en iguales condiciones laboratoriales ${ }^{[15]}$. En hospitales hindúes se halló cifras dispares: un $16,4 \%{ }^{[16]}$ y en otro un $62 \%{ }^{[17]}$. Estas diferencias se deberían a que en laboratorios de microbiología con recursos limitados sólo se aísla la cepa de mayor crecimiento y mayormente no se evalúan cepas anaerobias. El sistema automatizado Vytek, 
Tabla 6. Factores asociados a Enterobacterias BLEE (+) o SAMR en pie diabético infectado sin desenlace de amputación mayor del Hospital Nacional Edgardo Rebagliati Martins.

\begin{tabular}{|c|c|c|c|c|c|c|}
\hline \multirow{2}{*}{ Factores } & BLEE (+) & BLEE (-) & \multirow{2}{*}{ Valor de $p$} & SAMR (+) & SAMR (-) & \multirow{2}{*}{ - Valor de p } \\
\hline & $(n=26)$ & $(n=54)$ & & $(n=12)$ & $(n=5)$ & \\
\hline Edad (años \pm DE) & $58 \pm 13$ & $61 \pm 14$ & 0,47 & $59 \pm 10$ & $55 \pm 9$ & 0,38 \\
\hline Sexo masculino (n (\%)) & $24(92)$ & $45(83)$ & 0,27 & $8(66,7)$ & $3(60)$ & 0,06 \\
\hline Hemoglobina glicosilada (\% $₫ \mathrm{DE})$ & $8,5 \pm 2$ & $8,6 \pm 2$ & 0,85 & $9,3 \pm 2$ & $11 \pm 2$ & 0,23 \\
\hline Tiempo de diabetes (años $\pm D E$ ) & $20 \pm 9$ & $16 \pm 11$ & 0,10 & $14 \pm 9$ & $15 \pm 10$ & 0,88 \\
\hline Infección pie diabético previa. (n(\%)) & $14(54)$ & $8(15)$ & $<0,05$ & $9(75)$ & $2(40)$ & 0,16 \\
\hline Wagner $\geq 3$ (n (\%)) & $23(88)$ & $41(76)$ & 0,18 & $10(88)$ & $5(100)$ & 0,33 \\
\hline Isquemia moderada - severa. (n (\%)) & $19(76)$ & $40(75)$ & 0,42 & $7(58)$ & $3(60)$ & 0,48 \\
\hline IDSA moderado - severo ( $\mathrm{n}(\%))$ & $24(92)$ & $50(93)$ & 0,55 & $10(83)$ & $5(100)$ & 0,94 \\
\hline $\mathrm{PCR}(\mathrm{mg} / \mathrm{dL} \pm \mathrm{DE})$ & $12 \pm 9$ & $8 \pm 7$ & 0,04 & $13 \pm 6$ & $5 \pm 3$ & 0,01 \\
\hline Fascitis necrotizante $x$ LRINEC $\geq 8$ (n (\%)) & $8(35)$ & $8(17)$ & 0,04 & $3(38)$ & $0(0)$ & 0,10 \\
\hline
\end{tabular}

DE: Desviación estándar; IDSA: Infectious Diseases Society of America; LRINEC: Laboratory Risk Indicator for necrotizing fascitis; PCR: Proteína C reactiva.

utilizado en el presente trabajo, tiene una buena correlación con los métodos convencionales manuales, destacando la rapidez del resultado, brindando una potencial ayuda para mejorar el desenlace clínico de los pacientes ${ }^{[18]}$.

Las bacterias gramnegativas fueron las más frecuentes (69,5\%). De éstas, la $E$. coli fue la mayor en número $(23,4 \%)$ similar a la serie de Neyra ${ }^{[14]}$. Sin embargo, la serie de Elguera ${ }^{[15]}$ que evaluó el perfil bacteriológico 10 años antes en el mismo Hospital Loayza, encontró predominio de grampositivos. Series de la India ${ }^{[16,17,19,20]}$, Trinidad y Tobago ${ }^{[21]}$, Kuwait ${ }^{[22]}$, Túnez ${ }^{[23]}$ mostraron predominio de gramnegativos. Entre las Enterobacterias, las más frecuentes fueron E. coli ${ }^{[17,19,20]}$, Klebsiella sp. ${ }^{[21]}$ y Proteus sp. ${ }^{[22]}$; excepto en dos series en que predominó Pseudomonas sp. ${ }^{[16,22]}$. En cambio, series de Portugal ${ }^{[24]}$ y Pakistan ${ }^{[25]}$ muestran predominio de grampositivos. La mayor presencia gramnegativos se debería a una mayor colonización directa o indirecta por parte del personal de salud con déficit de lavado de manos, estancia prolongada, uso crónico de antibióticos, o mayor severidad de las lesiones ${ }^{[4,7]}$.

Los antibióticos con menor resistencia en bacterias gramnegativas fueron los carbapenémicos y amikacina. Los carbapenémicos también resultan ser los más sensibles en otras series peruanas, aunque evaluada de manera general ${ }^{[14,15]}$. Sin embargo los aminoglucósidos, no deben ser considerados en la terapéutica en pie diabético, ya que no tienen buena biodisponibilidad en tejido óseo.

El mecanismo de resistencia más importante de las bacterias gramnegativas a los betalactámicos es la producción de betalactamasas. Los genes que las codifican pueden encontrarse en el cromosoma bacteriano o en plásmidos y se producen de manera constitutiva o inducible; siendo las más importantes: las BLEE, las betalactamasas tipo AmpC y las carbapenemasas. Los carbapenémicos son útiles en el tratamiento de infecciones por enterobacterias productoras de BLEE y AmpC; sin embargo, la producción de carbapenemasas favorece su resistencia ${ }^{[8,26]}$. En nuestro estudio, $57 \%$ de las $E$. coli, el $40 \%$ de $K$. pneumoniae y el $31 \%$ de $P$. mirabilis fueron productoras de BLEE ( $33 \%$ de las todas las enterobacterias). Sólo una enterobacteria BLEE positiva presentó resistencia a un carbapenémico. Las otras series peruanas no evaluaron la presencia de BLEE ${ }^{[14,15]}$.

Para las enterobacterias no BLEE, cefepime y carbapenémicos mostraron las menores resistencias y los antibióticos usuales como ceftriaxona y ciprofloxacino presentaron resistencias del 56 y $69,4 \%$ respectivamente.

$P$. aeruginosa, bacteria gramnegativa no fermentadora, presentó una resistencia del $83 \%$ para imipenem y meropenem. Un reporte del INS halló una resistencia a imipenem del $28,5 \%$ y meropenem del $43,2 \%$ en todo de tipo de infección hospitalaria a nivel nacional ${ }^{[5]}$. Un estudio local halló una resistencia del $0 \%$ a imipenem ${ }^{[15]}$ en pie diabético. Otros fármacos de acción antipseudomona como ciprofloxacino y ceftazidima tuvieron una alta resistencia de $83 \%$ respectivamente y una baja resistencia a amikacina (17\%). Una serie local halló una resistencia de $70 \%$ para ciprofloxacino, $44 \%$ para ceftazidima y $45 \%$ para amikacina ${ }^{[14]}$. La impermeabilidad de membrana de $P$. aeruginosa, constituye uno de los mecanismos más significativos de resistencia frente a los carbapenémicos. Las alternativas terapéuticas frente a ello, se limitan al uso de antiguos antibióticos como la colistina ${ }^{[27]}$.

Otra bacteria gramnegativa no fermentadora con alta resistencia es el $A$. baumannii. De las 3 cepas halladas, todas tenían resistencia al $100 \%$ en todos los antibióticos. No obstante, esta extrema resistencia, los pacientes no culminaron en amputación mayor.

Al analizar los factores clínicos con la presencia o ausencia de $\mathrm{BLEE}$, se halló asociación con infección previa con pie diabético. El 
tratamiento crónico de las heridas podría ocasionar colonización con bacterias nosocomiales de alta resistencia. Una deficiente asepsia directa o indirecta por parte del personal asistencial y de la familia podría ocasionar infecciones de complicado tratamiento ${ }^{[4,7]}$. Además, un resultado más alto de PCR y una mayor proporción de fascitis necrotizante por LRINEC también se asociaron con BLEE (+).

Del total de bacterias, el E. faecalis representa el grampositivo con mayor porcentaje $(14,1 \%)$ seguido del $S$. aureus $(13,3 \%)$, similar a un estudio hindú ${ }^{[16]}$, pero en contra posición con todos los demás estudios que ubican a este último como el grampositivo más frecuente ${ }^{[14,17,19,20-22]}$. De todos los grampositivos, el $71 \%$ resultó ser SAMR, en concordancia con un reporte a nivel nacional del INS que encontró una frecuencia del $71,7 \%$ en pacientes hospitalizados por cualquier infección ${ }^{[5]}$. Un estudio local en pacientes con pie diabético infectado encontró una frecuencia de SAMR del $68 \%{ }^{[15]}$.

El mecanismo de resistencia a meticilina de $S$. aureus se asocia en general a la síntesis de una nueva proteína fijadora de penicilina (PBP2a ó PBP2) modificada o supernumeraria, conocida como resistencia intrínseca a meticilina ${ }^{[28]}$. Los factores de riesgo incluyen colonización previa, contacto con portadores de SAMR, exposición a una unidad/área con brote de SAMR, hemodiálisis, condiciones comórbidas, hospitalización, cirugía, admisión a unidad de cuidados intensivos y exposición a fluoroquinolonas. Los SAMR son un factor clave sobre los costes sanitarios, por la necesidad de tratamientos parenterales, prolongación de la estancia hospitalaria; además, el uso masivo y selectivo de glucopéptidos, puede llegar a desencadenar resistencia a este tipo de compuestos ${ }^{[29]}$. En los hospitales, el SAMR puede diseminarse de paciente a paciente, siendo el vehículo de transmisión el personal hospitalario y los objetos inanimados. El estado de portador puede persistir por años en algunos pacientes, garantizando una diseminación de estas cepas una vez que los pacientes vuelven a sus comunidades ${ }^{[15]}$.

Los SAMR presentaron también altas resistencias a ciprofloxacino (100\%) y clindamicina (89\%); pero bajas resistencias a rifampicina $(25 \%)$, cotrimoxazol $(33 \%)$ y vancomicina $(0 \%)$; similares resistencias también fueron observadas en otros estudios ${ }^{[6,15]}$. El uso de rifampicina en zonas de alta prevalencia de tuberculosis multidrogo-resistente, debe valorarse considerando el costobeneficio terapéutico ${ }^{[30]}$.

En S. aureus no meticilino resistente, la oxacilina es el medicamento de elección, levofloxacino, rifampicina y trimetoprim/sulfametoxazol también mostraron resistencias del $0 \%$, siendo alternativas orales eficaces para el manejo ambulatorio. El E. faecalis tuvo una baja resistencia a ampicilina (5\%) siendo el tratamiento de elección porque se puede continuar al alta en forma oral. No hubo resistencia a vancomicina ni en $S$. aureus, ni en $E$. faecalis.

Al analizar los factores clínicos con la presencia de SAMR, sólo se asoció con un resultado más alto de PCR, siendo este uno de los marcadores más precisos para detectar inflamación de causa infecciosa ${ }^{[9]}$. Debido a la baja frecuencia de $S$. aureus pudo no haberse encontrado asociación con más variables.
Muchos de los medicamentos de primera línea para terapia antibiótica empírica como la combinación ciprofloxacino/ clindamicina o ceftriaxona/clindamicina, presentan resistencias del $50 \%$ al $70 \%$. Por tanto, una combinación empírica adecuada para un paciente con un antecedente de infección por pie diabético, es el uso de un carbapenem asociado a vancomicina hasta el resultado del cultivo en 72 horas ${ }^{[31]}$. Además, para reducir la estancia hospitalaria, se considera el tratamiento antibiótico parenteral ambulatoria o domiciliaria que requiere: una minuciosa evaluación clínica del sujeto; selección adecuada del antimicrobiano incluyendo eficacia, vía, frecuencia, dosis, duración; vigilancia clínico-laboratorial de los efectos adversos y cuidado adecuado del acceso venoso ${ }^{[32]}$. Considerando que el ertapenem se puede usar una vez al día, por vía intramuscular/ endovenosa y no cubre a $P$. aeruginosa (bacteria que no es habitual en este tipo de infecciones), se podría considerar su uso en pacientes que no requieren hospitalización o para continuación de terapia al alta ${ }^{[31,32]}$.

La progresiva resistencia bacteriana a múltiples agentes antimicrobianos ha llevado a establecer consensos para nuevas definiciones de resistencia, que tomando como modelo las definiciones usadas para la tuberculosis: multidrogo-resistencia (MDR), extrema drogo-resistencia (XDR) y pandrogo-resistencia (PDR); determinarán también grados de susceptibilidad para las bacterias de mayor importancia clínica como el $S$. aureus, Enterococcus sp, Enterobacterias, P. aeruginosa y Acinetobacter $s p$. Aplicar estos conceptos ayudará a comparar y entender mejor el problema de la resistencia bacteriana ${ }^{[33]}$.

Como limitaciones del estudio consideramos la no inclusión de pacientes con amputación mayor para evaluar factores microbiológicos asociados a la pérdida/conservación de la extremidad inferior. También una pérdida de más del $50 \%$ del total de pacientes disponibles según criterios de inclusiónexclusión, debido a falta de datos completos. Otra limitación fue la no inclusión de cultivos anaerobios, ya que no se realizan rutinariamente en el nosocomio. Así mismo, una baja descripción de antibióticos ambulatorios previos, tiempo de utilización, tiempo de úlcera y hospitalizaciones previas para otras infecciones por no estar disponibles en la historia clínica.

Dentro de las fortalezas consideramos una descripción adecuada de grado de infección y compromiso vascular; la realización de técnica adecuada en toma de muestra de cultivo, como también de un sistema automatizado para la identificación bacteriana y de sensibilidad antimicrobiana.

Concluimos que en pacientes con pie diabético salvados de amputación mayor se encuentra una elevada frecuencia de enterobacterias BLEE positivas y SAMR. Infección previa, PCR alto y LRINEC $\geq 8$ estuvo asociado a la presencia de BLEE y sólo PCR alto a SAMR.

\section{Fuente de financiamiento: Autofinanciado}

Declaración de conflicto de interés: Los autores declaran no tener conflictos de interés en la publicación de este artículo. 


\section{REFERENCIAS BIBLIOGRÁFICAS}

1. Driver VR, Fabbi M, Lavery LA, Gibbons $G$. The costs of diabetic foot: the economic case for the limb salvage team. J Vasc Surg. 2010;52(3 Suppl):17S-22S.

2. Boulton AJ, Vileikyte L, Ragnarson-Tennvall G, Apelqvist J. The global burden of diabetic foot disease. Lancet. 2005;366(9498):1719-24.

3. Williams R, Airey M. The size of the problem: epidemiological and economic aspects of foot problems in diabetes. In: Boulton A, Cavanagh $\mathrm{P}$, Connor $\mathrm{H}$, editors. The foot in diabetes. 3rd ed. Chicester: John Wiley \& Sons; 2000. pp 3-17.

4. Lipsky BA, Berendt AR, Cornia PB, Pile JC, Peters EJ, Armstrong DG, et al. 2012 Infectious Diseases Society of America clinical practice guideline for the diagnosis and treatment of diabetic foot infections. Clin Infect Dis. 2012;54(12):e132-73.

5. Instituto Nacional de Salud. Informe de la resistencia antimicrobiana en hospitales en Perú - 2007 [Internet]. Lima: INS; 2007 [citado el 26 agosto 2015] Disponible en: http://www.ins.gob.pe/ repositorioaps/0/4/jer/-1/Informe_Resistencia_2007.pdf

6. Boucher HW, Talbot GH, Bradley JS, Edwards JE, Gilbert D, Rice LB, et al. Bad Bugs, No Drugs: No ESKAPE! An Update from the Infectious Diseases Society of America. Clin Infec Dis. 2009;48(1):1-12.

7. Lipsky BA, Aragón-Sánchez J, Diggle M, Embil J, Kono S, Lavery L, et al. IWGDF guidance on the diagnosis and management of foot infections in persons with diabetes. Diabetes Metab Res Rev. 2016 J; 32(Suppl 1):45-74.

8. Máttar S, Martínez P. Emergencia de la resistencia antibiótica debida a las ß-lactamasas de espectro extendido (BLEE): detección, impacto clínico y epidemiología. Infectio. 2007;11(1):23-35

9. Liao $\mathrm{CH}$, Lee $\mathrm{YK}$, Su YC, Chuang $\mathrm{CH}$, Wong $\mathrm{CH}$. Validation of the laboratory risk indicator for necrotizing fasciitis (LRINEC) score for early diagnosis of necrotizing fasciitis. Tzu Chi Medical Journal. 2012;24(2):73-6.

10. Torres-Aparcana H, Gutiérrez C, Pajuelo-Ramírez J, Pando-Alvarez R, Arbañil-Huamán H. Características clínicas y epidemiológicas de los pacientes hospitalizados por pie diabético en el Hospital Nacional Dos de Mayo entre 2006 y 2008, Lima-Perú. Rev Peru Epidemiol. 2012;16(3):1-6.

11. Alcantara W, Flores R, Garmendia F. Prevalencia y riesgo de amputación en pacientes con pie diabético. An Fac Med. 1999;60(3):159.

12. Damas-Casani V, Yovera-Aldana M, Seclén S. Clasificación de pie en riesgo de ulceración según el Sistema IWGDF y factores asociados en pacientes con diabetes mellitus tipo 2 de un hospital peruano. Rev Med Hered. 2017;28(1):5-12.

13. Escalante D, Lecca L, Gamarra J, Escalante G. Amputación del miembro inferior por pie diabético en hospitales de la costa norte peruana 1990 - 2000: características clínico-epidemiológicas. Rev Peru Med Exp Salud Publica. 2003;20(3):138-44.

14. Neyra L, Lezama L, Lezama W, Medina M, Murillo F, Purizaga H, et al. Bacteriología y resistencia bacteriana en el pie diabético infectado en el Hospital Nacional Arzobispo Loayza, Lima. Rev Soc Peru Med Interna. 2017;30(1):24-7.

15. Elguera F, Solíz J, Neyra L. Estudio bacteriológico de pacientes con pie diabético infectado en el Hospital Arzobispo Loayza. Rev Soc Med Inter. 2006;19(1):5-9.

16. Turhan V, Mutluoglu M, Acar A, Hatipoglu M, Onem Y, Uzun G, et al. Increasing incidence of Gram-negative organisms in bacterial agents isolated from diabetic foot ulcers. J Infect Dev Ctries. 2013;7(10):70712.

17. Zubair M, Malik A, Ahmad J. Clinico-microbiological study and antimicrobial drug resistance profile of diabetic foot infections in North India. Foot (Edinb). 2011;21(1):6-14.
18. Vargas LJ, Vila A, Lanza A, Bonvehi P, Nazar J, Mikietuk A, et al. Utilidad del sistema VITEK en la identificación bacteriana y estudios de sensibilidad antimicrobiana. Acta Bioquim Clin Latinoam. 2005;39(1):19-25.

19. Tiwari S, Pratyush DD, Dwivedi A, Gupta SK, Rai M, Singh SK. Microbiological and clinical characteristics of diabetic foot infections in northern India. J Infect Dev Ctries. 2012;6(4):329-32.

20. Parvez N, Dutta P, Ray P, Shah VN, Prakash M, Khandelwal N, et al. Microbial profile and utility of soft tissue, pus, and bone cultures in diagnosing diabetic foot infections. Diabetes Technol Ther 2012;14(8):669-74.

21. Islam S, Cawich SO, Budhooram S, Harnarayan P, Mahabir V, Ramsewak $S$, et al. Microbial profile of diabetic foot infections in Trinidad and Tobago. Prim Care Diabetes. 2013;7(4):303-8.

22. Benwan KA, Mulla AA, Rotimi VO. A study of the microbiology of diabetic foot infections in a teaching hospital in Kuwait. J Infect Public Health. 2012;5(1):1-8.

23. Moussa MB, Khalfallah M, Boubaker IBB, Nouira R, Slim A, Jerraya H, et al. Profil bactériologique et thérapeutique du pied diabétique infecté étude prospective de 100 patients. Tunis Med. 2016;94(2):95-101.

24. Mendes JJ, Marques-Costa A, Vilela C, Neves J, Candeias N, CavacoSilva $\mathrm{P}$, et al. Clinical and bacteriological survey of diabetic foot infections in Lisbon. Diabetes Res Clin Pract. 2012;95(1):153-61.

25. Aamir AH, Nasir A, Jadoon MZ, Mehmood K, Ali SS. Diabetic foot infections and their management in a tertiary care hospital. J Ayub Med Coll Abbottabad. 2011;23(1):58-62.

26. García T, Castillo A, Salazar D. Mecanismos de resistencia a betalactámicos en bacterias gramnegativas. Rev Cubana Salud Publica. 2014;40(1):129-35.

27. Fica C, Alberto, Céspedes J, Ignacio, Gompertz G, Macarena, et al. Colistín en infecciones nosocomiales por bacilos gramnegativos panresistentes. Rev Chil Infectol. 2007;24(5):360-7.

28. Camarena JJ, Sánchez R. infección por staphylococcus aureus resistente a meticilina [Internet]. Madrid: Sociedad Española de Enfermedades Infecciosas y Microbiología Clínica; 1999 [citado el 26 junio 2017]. Disponible en: https://www.seimc.org/contenidos/ccs/ revisionestematicas/bacteriologia/sarm.pdf

29. Domínguez M, Pujol M. Cambios en la epidemiología de staphylococcus aureus resistente a la meticilina. Recomendaciones para el control de su diseminación[Internet]. Madrid: Sociedad Española de Enfermedades Infecciosas y Microbiología Clínica; 2006 [citado el 26 junio 2017]. Disponible en: https://www.seimc.org/contenidos/ccs/ revisionestematicas/bacteriologia/Marsactrl.pdf

30. De la Iglesia Al, Morbidoni HR. Mecanismos de acción y de resistencia a rifampicina e isoniacida en Mycobacterium tuberculosis: nueva información sobre viejos conocidos. Rev Argent Microbiol. 2006;38(2):97-109.

31. Crouzet J, Lavigne JP, Richard JL, Sotto A. Diabetic foot infection: a critical review of recent randomized clinical trials on antibiotic therapy. Int J Infect Dis. 2011;15(9):e601-10.

32. Alavi A, Bader M, Sibbald R. Management of diabetic foot infections with appropriate use of antimicrobial therapy. Clin Res Foot Ankle [Internet]. 2014 [Citado el 25 junio 2017]; S3:010. Disponible en: http://www.esciencecentral.org/journals/management-of-diabeticfoot-infections-with-appropriate-use-of-antimicrobial-therapy-2329910X-S3-010.pdf

33. Magiorakos AP, Srinivasan A, Carey RB, Carmeli Y, Falagas ME, Giske CG, et al. Multidrug-resistant, extensively drug-resistant and pandrugresistant bacteria: an international expert proposal for interim standard definitions for acquired resistance. Clin Microbiol Infect. 2012;18(3):268-81. 